\title{
Rapidity Gap Survival in the Black-Disk Regime
}

\author{
L. Frankfurt ${ }^{1}$, C.E. Hyde ${ }^{2}$, M. Strikman ${ }^{3}$, C. Weiss ${ }^{4}$ * \\ 1- School of Physics and Astronomy, Tel Aviv University, Tel Aviv, Israel \\ 2- Old Dominion University, Norfolk, VA 23529, USA, \\ and Laboratoire de Physique Corpusculaire, Université Blaise Pascal, 63177 Aubière, France \\ 3- Department of Physics, Pennsylvania State University, University Park, PA 16802, USA \\ 4- Theory Center, Jefferson Lab, Newport News, VA 23606, USA
}

\begin{abstract}
We summarize how the approach to the black-disk regime (BDR) of strong interactions at $\mathrm{TeV}$ energies influences rapidity gap survival in exclusive hard diffraction $p p \rightarrow p+H+p(H=\operatorname{dijet}, \bar{Q} Q$, Higgs $)$. Employing a recently developed partonic description of such processes, we discuss (a) the suppression of diffraction at small impact parameters by soft spectator interactions in the BDR; (b) further suppression by inelastic interactions of hard spectator partons in the BDR; (c) correlations between hard and soft interactions. Hard spectator interactions substantially reduce the rapidity gap survival probability at LHC energies compared to previously reported estimates.
\end{abstract}

\section{Introduction}

At high energies strong interactions enter a regime in which cross sections are comparable to the "geometric size" of the hadrons, and unitarity becomes an essential feature of the dynamics. By analogy with quantum-mechanical scattering from a black disk, in which particles with impact parameters $b<R_{\text {disk }}$ experience inelastic interactions with unit probability, this is known as the black-disk regime (BDR). The approach to the BDR is well-known in soft interactions, where it generally can be attributed to the "complexity" of the hadronic wave functions. It is seen e.g. in phenomenological parametrizations of the $p p$ elastic scattering amplitude, whose profile function $\Gamma(b)$ approaches unity at $b=0$ for energies $\sqrt{s} \gtrsim 2 \mathrm{TeV}$. More recently it was realized that the BDR is attained also in hard processes described by QCD, due to the increase of the gluon density in the proton at small $x$. Theoretically, this phenomenon can be studied in the scattering of a small-size color dipole $(d \sim 1 / Q)$ from the proton. Numerical studies show that that at $\mathrm{TeV}$ energies the dipole-proton interaction is close to "black" up to $Q^{2} \sim$ several $10 \mathrm{GeV}^{2}$ [2]. This fact has numerous implications for the dynamics of $p p$ collisions at the LHC, where multiple hard interactions are commonplace. For example, it predicts dramatic changes in the multiplicities and $p_{T}$ spectra of forward particles in central $p p$ collisions compared to extrapolations of the Tevatron data [3]. Absorption and energy loss of leading partons by inelastic interactions in the BDR can also account for the pattern of forward pion production in $d-A u$ collisions at STAR [4].

Particularly interesting is the question what the approach to the BDR implies for exclusive hard diffractive scattering, $p p \rightarrow p+H+p$. In such processes a high-mass system ( $H=$ dijet, $\bar{Q} Q$, Higgs) is produced in a hard process involving exchange of two gluons between the protons. At the same time, the spectator systems must interact in a way such as not to produce additional particles. This restricts the set of possible trajectories in configuration space and results in a suppression of the cross section compared to non-diffractive

\footnotetext{
*Notice: Authored by Jefferson Science Associates, LLC under U.S. DOE Contract No. DE-AC0506OR23177. The U.S. Government retains a non-exclusive, paid-up, irrevocable, world-wide license to publish or reproduce this manuscript for U.S. Government purposes.
} 


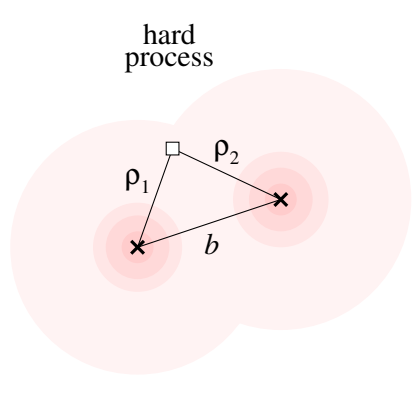

(a)

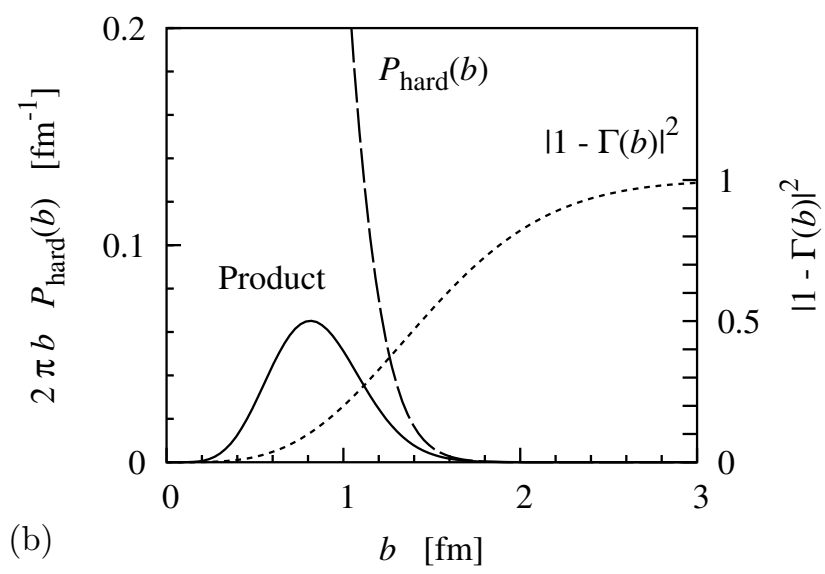

(b)

Figure 1: (a) Transverse geometry of hard diffractive $p p$ scattering. (b) Dashed line: Probability for hard scattering process $P_{\text {hard }}(\boldsymbol{b})$ as function of the $p p$ impact parameter, $b$. Dotted line: Probability for no inelastic interactions between the protons, $|1-\Gamma(\boldsymbol{b})|^{2}$. Solid line: Product $P_{\text {hard }}(\boldsymbol{b})|1-\Gamma(\boldsymbol{b})|^{2}$. The RGS probability (1) is given by the area under this curve. The results shown are for Higgs production at the LHC $\left(\sqrt{s}=14 \mathrm{TeV}, M_{H} \sim 100 \mathrm{GeV}\right)$.

events. For soft spectator interactions this suppression is measured by the so-called rapidity gap survival (RGS) probability. Important questions are (a) what role the BDR plays in traditional soft-interaction RGS; (b) how the physical picture of RGS is modified by hard spectator interactions in the BDR at LHC energies; (c) how possible correlations between hard and soft interactions affect RGS. These questions can be addressed in a recently proposed partonic description of exclusive diffraction [5], based on Gribov's parton picture of high-energy hadron-hadron scattering. This approach allows for a model-independent treatment of the interplay of hard and soft interactions, and for the consistent implementation of the BDR at high energies (for details, see Ref. [5]).

\section{Black-disk regime in soft spectator interactions}

A simple "geometric" picture of RGS is obtained in the approach of Ref. [5] in the approximation where hard and soft interactions are considered to be independent. The hard two-gluon exchange process can be regarded as happening locally in space-time on the typical scale of soft interactions. In the impact parameter representation (see Fig. 1a) the RGS probability can be expressed as

$$
S^{2}=\int d^{2} b P_{\text {hard }}(\boldsymbol{b})|1-\Gamma(\boldsymbol{b})|^{2} .
$$

Here $P_{\text {hard }}(\boldsymbol{b})$ is the probability for two hard gluons from the protons to collide in the same space-time point, given by the overlap integral of the squared transverse spatial distributions of gluons in the colliding protons, normalized to $\int d^{2} b P_{\text {hard }}(\boldsymbol{b})=1$ (see Fig. 1b). The function $|1-\Gamma(\boldsymbol{b})|^{2}$ is the probability for the two protons not to interact inelastically in a collision with impact parameter $b$. The approach to the BDR in $p p$ scattering at energies 


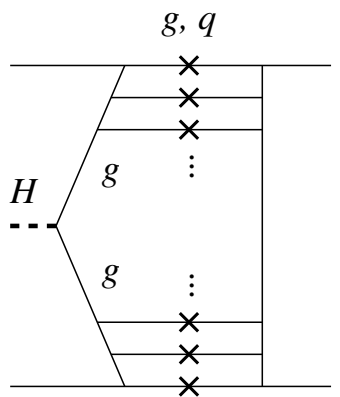

$g, q$

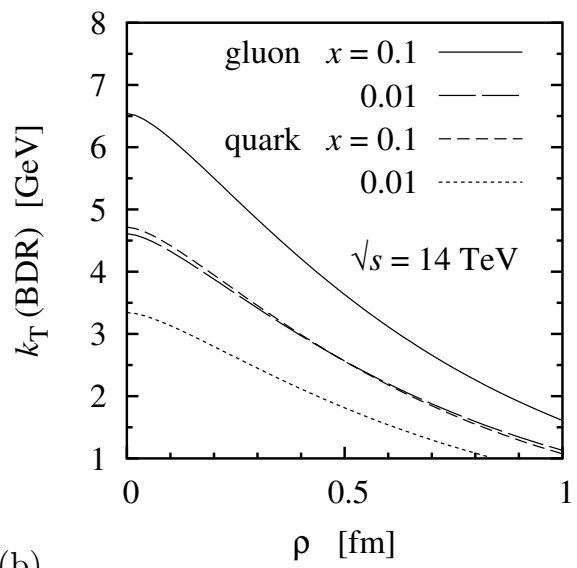

(a)

Figure 2: (a) Absorption of parent partons in the evolution by interactions in the BDR. (b) The critical transverse momentum, $k_{T}(\mathrm{BDR})$, below which partons are absorbed with high probability $\left(\left|\Gamma^{\text {parton-proton }}\right|>0.5\right)$, as a function of the parton-proton impact parameter, $\rho$.

$\sqrt{s} \gtrsim 2 \mathrm{TeV}$ implies that this probability is practically zero at small impact parameters, and becomes significant only for $b \gtrsim 1 \mathrm{fm}$ (see Fig. 1b). This eliminates the contribution from small impact parameters in the integral (1) (see Fig. 1b) and determines the value of the RGS probability to be $S^{2} \ll 1$. It is seen that the approach to the BDR in soft interactions plays an essential role in the physical mechanism of RGS.

\section{Black-disk regime in hard spectator interactions}

At LHC energies even highly virtual partons $\left(k^{2} \sim\right.$ few $\left.\mathrm{GeV}^{2}\right)$ with $x \gtrsim 10^{-2}$ experience "black" interactions with the small- $x$ gluons in the other proton. This new effect causes an additional suppression of diffractive scattering which is not included in the traditional RGS probability [5]. One mechanism by which this happens is the absorption of "parent" partons in the QCD evolution leading up to the hard scattering process (see Fig. 2a). Specifically, in Higgs production at the LHC the gluons producing the Higgs have momentum fractions $x_{1,2} \sim M_{H} / \sqrt{s} \sim 10^{-2}$; their "parent" partons in the evolution (quarks and gluons) typically have momentum fractions of the order $x \sim 10^{-1}$ and transverse momenta $k_{T}^{2} \sim$ few $\mathrm{GeV}^{2}$. Quantitative studies of the BDR in the dipole picture show that at the LHC energy such partons are absorbed with near-unit probability if their impact parameters with the other proton are $\rho_{1,2} \lesssim 1 \mathrm{fm}$ (see Fig. $2 \mathrm{~b}$ ). For proton-proton impact parameters $b<1 \mathrm{fm}$ about $90 \%$ of the strength in $P_{\text {hard }}(b)$ comes from parton-proton impact parameters $\rho_{1,2}<1 \mathrm{fm}$ (cf. Fig. 1a), so that this effect practically eliminates diffraction at $b<1 \mathrm{fm}$. Since $b<1 \mathrm{fm}$ accounts for $2 / 3$ of the cross section [see Eq. (1) and Fig. 1b)], and the remaining contributions at $b>1 \mathrm{fm}$ are also reduced by absorption, we estimate that inelastic interactions of hard spectators in the BDR reduce the RGS probability at LHC energies to about $20 \%$ of its soft-interaction value. (Trajectories with no emissions, corresponding to the $\delta(1-x)$ term in the evolution kernel, are not affected by absorption; however, their contributions 
are small because they effectively probe the gluon density at the soft input scale.) Since this effect "pushes" diffractive $p p$ scattering to even larger impact parameters than allowed by soft-interaction RGS it should also manifest itself in a shift of the final-state proton transverse momentum distribution to smaller values, which could be observed in $p_{T}$-dependent measurements of diffraction at the LHC.

The estimates reported here are based on the assumption that DGLAP evolution reasonably well describes the gluon density down to $x \sim 10^{-6}$; the quantitative details (but not the basic picture) may change if small- $x$ resummation corrections were to significantly modify the gluon density at such values of $x$ (see Ref. [6] and references therein). The effect of hard spectator interactions described here is substantially weaker at the Tevatron energy.

\section{Correlations between hard and soft interactions}

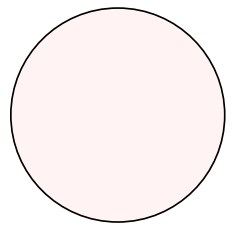

uncorrelated

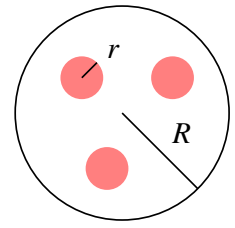

correlated

Figure 3: Transverse parton correlations.

The partonic approach to RGS of Ref. [5] also allows one to incorporate effects of correlations in the partonic wavefunction of the protons. They can lead to correlations between hard and soft interactions in diffraction, which substantially modify the picture of RGS compared to the independent interaction approximation. The CDF data on $p p$ collisions with multiple hard processes indicate the presence of substantial transverse correlations of size $r \ll R$ between partons (see Fig. 3). A possible explanation of their origin could be the "constituent quark" structure of the proton suggested by the instanton vacuum model of chiral symmetry breaking. Such correlations modify the picture of RGS in hard diffractive $p p$ scattering compared to the independent interaction approximation in two ways [5]. On one hand, with correlations inelastic interactions between spectators are much more likely in configurations in which two large $-x$ partons collide in a hard process than in average configurations, reducing the RGS probability compared to the uncorrelated case. On the other hand, the "lumpiness" implies that there is generally a higher chance for the remaining spectator system not to interact inelastically compared to the mean-field approximation. A quantitative treatment of correlations in RGS, incorporating both effects, remains an outstanding problem.

\section{References}

[1] Slides: http: //indico. cern. ch/contributionDisplay. py? contribId=67\&sessionId=7\& conf Id=9499

[2] For a review, see: L. Frankfurt, M. Strikman and C. Weiss, Ann. Rev. Nucl. Part. Sci. 55, 403 (2005).

[3] L. Frankfurt, M. Strikman and C. Weiss, Phys. Rev. D 69, 114010 (2004).

[4] L. Frankfurt and M. Strikman, Phys. Lett. B 645, 412 (2007).

[5] L. Frankfurt, C. E. Hyde-Wright, M. Strikman and C. Weiss, Phys. Rev. D 75, 054009 (2007).

[6] M. Ciafaloni, D. Colferai, G. P. Salam and A. M. Stasto, arXiv:0707.1453 [hep-ph]. 